\title{
Editorial
}

\section{O Congresso Brasileiro de Psicologia: Ciência e Profissão como um lócus privilegiado de comunicação científica}

\author{
Rodolfo Augusto Matteo Ambiel \\ Ana Paula Porto Noronba \\ Lucas de Francisco Carvalho \\ Universidade São Francisco - Campinas, SP, Brasil
}

A cada quatro anos ocorre um importante evento científico no Brasil, o Congresso Brasileiro de Psicologia: Ciência e Profissão. Chamado pelo apelido de "Congressão", em 2018 aconteceu a quinta edição desse evento, entre 14 e 17 de novembro, na cidade de São Paulo. Este é o maior evento científico da Psicologia brasileira, com mais de 14.000 inscritos, dos quais, estima-se que aproximadamente $70 \%$ sejam estudantes de graduação.

Outra característica bastante particular do Congressão é sua pluralidade de temas. O evento é organizado pelo Fórum de Entidades Nacionais da Psicologia Brasileira (FENPB) que em 2018 congregou 26 entidades profissionais e científicas, das mais variadas áreas de atuação e pesquisa da Psicologia. Nesse sentido, e possível inferir que os quase 6000 trabalhos aprovados para apresentação durante o congresso contam também com uma infinidade de temas e metodologias e acabam se tornando uma fonte importante de formação para o Psicólogo brasileiro.

É importante grifar que a modalidade de comunicação científica por meio de trabalhos em congressos facilita um divulgação direta e interativa entre o autor e o público interessado. Apesar da imensa quantidade de trabalhos aprovados, a lógica em um evento como esse está muito distante do cenário desalentador comentado por Rego (2014), no qual impera o produtivismo acadêmico, ou mesmo dos achados de Mugnaini, Digiampietri e Mena-Chalco (2014), que relatam uma tendência cada vez maior da disseminação do conhecimento das ciências humanas e sociais em revistas estrangeiras e, em geral, em língua inglesa. Em um congresso, especialmente um do tamanho e com as características do Congressão, há a divulgação de conhecimento predominantemente produzido na base, muitas vezes dentro dos cursos de graduação, por estudantes ávidos por desenvolvimento e por encontros pessoais e acadêmicos que somente um congresso científico pode proporcionar.
Por outros meios, a revista Psico-USF também busca aproximar os leitores dos autores e aqui apresenta o quarto número de 2018 . Nesse volume 23 , número 4, são oferecidos 14 artigos inéditos e uma resenha que visam contribuir para a ciência da Psicologia. Esperamos que nossos leitores desfrutem desses trabalhos e possam adquirir novos conhecimentos.

O primeiro trabalho apresentado nesta edição se refere à Reading Comprehension and Self-Perceived School Performance in Elementary School, de autoria de Andrea Carvalho Beluce, Amanda Lays Monteiro Inácio, Katya Luciane de Oliveira e Sandra Aparecida Pires Franco. As autoras tiveram por objetivo investigar, em alunos do ensino fundamental, a compreensão em leitura e possíveis associações com o desempenho escolar autopercebido.

Hannah D. Haemer e Jairo E. Borges-Andrade realizaram uma pesquisa com o intuito de reunir evidências de validade para a Escala de Estratégias de Aprendizagem no trabalho. Esse artigo foi intitulado Learning Strategies at Work Scale: a Confirmatory Factor Analysis.

The Structural Approach to Social Representations: Bridges between Theory and Methods é um artigo teórico que apresenta as características das diferentes técnicas e métodos da abordagem estrutural das representações sociais. A autoria é de Rafael Wolter.

O foco da revisão de literatura de Larissa Sanford Ayres Farina, Gabriel dos Reis Rodrigues e Claudio Simon Hutz foi investigar as relações entre engajamento no trabalho e flow, além de apresentar as principais conceituações a respeito deles. O título do artigo é Flow and Engagement at Work: a Literature Review.

Naiana Dapieve Patias, Cíntia Debon, Sibeli Carla Garbin Zanin e Aline Cardoso Siqueira realizaram a pesquisa intitulada How have parents raised their kids? Adolescent's perception of parental responsiveness and demandingness. As autoras investigaram o estilo parental adotado pelos cuidadores dos adolescentes a partir do 
cruzamento das dimensões exigência e responsividade, como também por sexo e faixa etária.

Processos Identitários de um Grupo de Doulas: Atitudes sobre Gestantes e Médicos é um estudo de Camila Nogueira Bonfim Duarte e Luiz Gustavo Silva Souza. Os autores investigaram os processos identitários de um grupo de doulas, incluindo a percepção que essas tinham sobre o próprio grupo, medicos e gestantes.

O objetivo dos autores Fernanda Andrade de Freitas Salgado, Soely Aparecida Jorge Polydoro e Pedro Sales Luis da Fonseca Rosário foi avaliar a eficácia de um programa de promoção da autorregulação da aprendizagem na educação superior. $\mathrm{O}$ artigo foi intitulado Programa de Promoção da Autorregulação da Aprendizagem de Ingressantes da Educação Superior.

A revisão de literatura narrativa intitulada Adaptação Cultural de Intervenções Preventivas em Saúde Mental Baseadas em Evidências é de autoria de Jordana Calil Lopes de Menezes e Sheila Giardini Murta. As autoras analisaram as etapas e características dos modelos de adaptação cultural de intervenções preventivas importadas (Planet Intervention Adaptation, Protocol Strengthening Families Program Adaptation Model e ADAPT-ITT).

As contribuições de Camélia Santina Murgo, Leonardo de Oliveira Barros e Bárbara Cristina Soares Sena podem ser consultadas no artigo Associações entre Estilos Parentais, Interesses e Indecisão Profissional em Estudantes do Ensino Médio. Os autores tiveram o intuito de verificar as relações entre estilos parentais, interesses profissionais e indecisão de adolescentes.

O estudo de caso sistemático Manifestações Contratransferenciais no Processo Terapêutico de uma Paciente com Personalidade Borderline é de autoria de Rochele Luciane Möller, Fernanda Barcellos Serralta, Aline Alvares Bittencourt e Silvia Pereira da Cruz Benetti. As autoras objetivaram descrever as manifestações contratransferenciais no processo terapêutico do primeiro ano de uma terapia psicanálitica de uma paciente com transtorno de personalidade borderline.

No estudo Racial Caracterização de Fatores de Risco para o Comportamento Criminal em Detentos o objetivo foi caracterizar fatores de risco, especificamente, a psicopatia, agressividade e a personalidade, em detentos brasileiros inseridos em atividade de ressocialização. O trabalho é de autoria de Germano Gabriel Lima Esteves, Jorge Artur Peçanha de Miranda Coelho, Bruna Nogueira Romariz Barros e Gustavo Henrique Silva de Souza.

Fernanda Palhares, Lia Beatriz de Lucca Freitas, Doralúcia Gil da Silva e Claudia Hofheinz Giacomoni realizaram a pesquisa intitulada Adolescentes Materialistas Brasileiros Estão Satisfeitos com suas Vidas? As autoras investigaram os níveis de materialism e suas relações com os níveis de satisfação de vida em adolescents de escolas privadas e públicas.

Pedir Desculpas: Categorias e Efeitos em Três Tipos de Relacionamento Interpessoal é de autoria de Vithor Rosa Franco, Fabio Iglesias e Izabella Rodrigues Melo. O objetivo investigar quais os principais tipos de desculpas que as pessoas utilizam e como são recebidas em três tipos de relacionamento interpessoal: com um parente íntimo; com um amigo íntimo; ou com um par romântico.

Juliana Célia de Oliveira e Altemir José Gonçalves Barbosa realizaram a pesquisa Saúde e Invariância Fatorial e Normatização das Escalas de Sobre-Excitabilidade. O objetivo dos autores foi estabelecer normas preliminaries e verificar a estrutura e invariância factorial das Escalas de Sobre-Excitabilidade de acordo com o sexo.

Por fim, é apresentada a resenha de Rodrigo da Silva Maia, intitulada Revisitando a segunda edição do livro "Neuropsicologia Hoje". A equipe da Psico-USF deseja que os leitores possam, por meio dessa edição, encontrar uma ferramenta valiosa para adquirir novos e significativos conhecimentos.

\section{Referências}

Mugnaini, R., Digiampietri, L. A., \& Mena-Chalco, J. P. (2014). Comunicação científica no Brasil (1998-2012): indexação, crescimento, fluxo e dispersão. TransInformação, 26(3), 239-252. Doi: 10.1590/0103-37862014000300002

Rego, T. C. (2014). Produtivismo, pesquisa e comunicação científica: entre o veneno e o remédio. Educação e Pesquisa, 40(2), 325-346. Doi: 10.1590/ S1517-97022014061843 